\title{
Protection by Pyruvate against Transient Forebrain Ischemia in Rats
}

\author{
Joo-Yong Lee, Yang-Hee Kim, and Jae-Young Koh \\ National Creative Research Initiative Center for the Study of CNS Zinc and Department of Neurology, University of Ulsan \\ College of Medicine, Seoul 138-736, Korea
}

Pyruvate has a remarkable protective effect against zinc neurotoxicity. Because zinc neurotoxicity is likely one of the key mechanisms of ischemic brain injury, the neuroprotective effect of pyruvate was tested in a rat model of transient forebrain ischemia. Control experiments in mouse cortical culture showed that pyruvate almost completely blocked zinc toxicity but did not attenuate calcium-overload neuronal death. Adult rats subjected to $12 \mathrm{~min}$ forebrain ischemia exhibited widespread zinc accumulation and neuronal death throughout hippocampus and cortex $72 \mathrm{hr}$ after reperfusion. However, rats injected intraperitoneally with sodium pyruvate (500-1000 mg/ $\mathrm{kg}$ ) within $1 \mathrm{hr}$ after 12 min forebrain ischemia showed almost no neuronal death. In addition, the mortality was markedly decreased in the pyruvate-protected groups (3.8\%) compared with the $\mathrm{NaCl}$-injected control group (58.1\%). The neuroprotective effect persisted even at $30 \mathrm{~d}$ after the insult. The spectacular protection without noticeable side effects makes pyruvate a promising neuroprotectant in human ischemic stroke.

Key words: zinc neurotoxicity; glycolysis; excitotoxicity; glutamate antagonist; stroke; ATP; NAD ${ }^{+}$
Neurons in the forebrain are highly vulnerable to ischemia (Pulsinelli et al., 1982; Smith et al., 1984). Only a few minutes of ischemia can cause death of vulnerable neurons in hippocampus and neocortex. Neuronal death in ischemic conditions may be brought about by a chain of events (failed energy metabolism, membrane depolarization, glutamate release, and toxic calcium influx) that comprise the vicious circle of calcium excitotoxicity (Choi and Rothman, 1990; Choi, 1995).

In addition to calcium, endogenous zinc may play a role as an ionic mediator of neuronal cell death (Choi and Koh, 1998; Weiss et al., 2000). Forebrain contains a substantial pool of chelatable zinc inside vesicles of certain excitatory synaptic boutons (Frederickson, 1989; Palmiter et al., 1996; Wenzel et al., 1997) that is released with neuronal activity or membrane depolarization (Assaf and Chung, 1984; Howell et al., 1984; Aniksztejn et al., 1987; Vogt et al., 2000). After brain ischemia, concurrent with depletion of presynaptic bouton zinc, accumulation of zinc in the cell body of vulnerable neurons occurs (Tonder et al., 1990; Koh et al., 1996). Furthermore, blockade of the zinc movement with the metal chelator CaEDTA, markedly reduces ischemic neuronal death (Koh et al., 1996; Park et al., 2000). These results suggest that the interneuronal movement of zinc is a key mechanism of ischemic neuronal death.

Zinc can enter neurons via various $\mathrm{Ca}^{2+}$ routes, such as NMDA channels, $\mathrm{Ca}^{2+}$-permeable AMPA/kainate channels, voltage-gated $\mathrm{Ca}^{2+}$ channels, and $\mathrm{Na}^{+} / \mathrm{Ca}^{2+}$ exchangers (Choi and Koh, 1998; Weiss et al., 2000), which are activated by coreleased glutamate. To highlight the fact that endogenous zinc, in addition to or instead of $\mathrm{Ca}^{2+}$, can mediate glutamate neurotox-

\footnotetext{
Received May 21, 2001; revised July 10, 2001; accepted July 19, 2001.

This work was supported by Creative Research Initiatives of the Korean Ministry of Science and Technology (J.-Y.K.)

Correspondence should be addressed to Jae-Young Koh, National Creative Research Initiative Center for the Study of CNS Zinc, Department of Neurology, University of Ulsan College of Medicine, 388-1 Poongnap-Dong Songpa-Gu, Seoul 138-736, Korea. E-mail: jkko@www.amc.seoul.kr.

Copyright (C) 2001 Society for Neuroscience $0270-6474 / 01 / 210001-06 \$ 15.00 / 0$
}

icity in certain cases, Frederickson et al (2000) coined the term "zinc excitotoxicity" as opposed to conventional "calcium excitotoxicity." Inside neurons, zinc may activate various cell death cascades, such as free radical generation and caspase activation (Kim et al., 1999; Lobner et al., 2000; Park et al., 2000).

As in brain ischemia, zinc neurotoxicity in cortical culture involves disturbances in energy metabolism. Sheline et al. (2000) showed that exposure to zinc causes inhibition of glyceraldehyde3-phosphate dehydrogenase and depletion of ATP and NAD ${ }^{+}$. Surprisingly, pyruvate, the end metabolite of glycolysis, normalizes depletion of ATP and NAD ${ }^{+}$and protects almost completely against zinc toxicity (Sheline et al., 2000).

If the neuroprotective effect of pyruvate is as remarkable in animal models of brain ischemia as in zinc neurotoxicity in cortical culture, pyruvate may be an ideal neuroprotectant in human ischemic stroke. To examine this, we tested the protective effect of pyruvate in a rat model of transient forebrain ischemia. Here we report that intraperitoneal pyruvate given within $1 \mathrm{hr}$ after the reperfusion almost completely blocks neuronal injury in this model.

\section{MATERIALS AND METHODS}

Cortical cell culture and exposure to toxins. Mixed cortical cell cultures, containing both neurons and astrocytes, were prepared as described

This article is published in The Journal of Neuroscience, Rapid Communications Section, which publishes brief, peerreviewed papers online, not in print. Rapid Communications are posted online approximately one month earlier than they would appear if printed. They are listed in the Table of Contents of the next open issue of JNeurosci. Cite this article as: JNeurosci, 2001, 21:RC171 (1-6). The publication date is the date of posting online at www.jneurosci.org.

http://www.jneurosci.org/cgi/content/full/5693 
previously (Koh et al., 1996; Kim et al., 1999) from fetal mice at 14-15 $\mathrm{d}$ of gestation. Briefly, dissociated cortical cells were plated onto a previously established astroglial cell monolayer at three hemispheres per 24-well plate (Nunc, Rochester, NY) in a plating medium [DMEM (Life Technologies, Rockville, MD) supplemented with $20 \mathrm{~mm}$ glucose, $38 \mathrm{~mm}$ sodium bicarbonate, $2 \mathrm{~mm}$ glutamine, $5 \%$ fetal bovine serum, and 5\% horse serum]. Cytosine arabinoside $(10 \mu \mathrm{M})$ was added 5-6 d after plating to halt the growth of non-neuronal cells. Glial cultures were prepared from neocortices of newborn mice (postnatal day 1-3) and plated at 0.5 hemispheres per 24-well plate, in the same plating medium but supplemented with $10 \%$ fetal bovine serum and $10 \%$ horse serum. Astroglial cultures were used for plating between 14 and $28 \mathrm{~d}$ in vitro, when they form a confluent monolayer. Continuous exposure to toxins (zinc as $\mathrm{ZnCl}_{2}$, NMDA, glutamate, and ionomycin) without or with an addition of $5 \mathrm{~mm}$ pyruvate (Sigma, St. Louis, MO) was done in MEM. After $24 \mathrm{hr}$ of the exposure onset, neuronal death was estimated.

Transient cerebral ischemia of the rat. All animal experiments were performed in accordance with the Guide of Ulsan University for Care and Use of Laboratory Animals. Male adult Sprague Dawley rats weighing 290-310 gm were used for experiments. Under halothane anesthesia, both common carotid arteries were ligated for $12 \mathrm{~min}$. At the same time, systemic mean arterial pressure was lowered to $50 \pm 5 \mathrm{mmHg}$ by withdrawing blood from femoral artery into heparinized syringe. Core body temperature was maintained at $37 \pm 1.0^{\circ} \mathrm{C}$ using a heating blanket (Harvard, South Natick, MA) and lamp during and for $2 \mathrm{hr}$ after the ischemia. Perfusion was restored by unligating the arteries and reintroducing the blood.

Sodium pyruvate $(500 \mathrm{mg} / \mathrm{kg})$ dissolved in water was given to rats by intraperitoneal injection at various times as indicated. As controls, osmolarity-matched $\mathrm{NaCl}(104.5 \mathrm{mg} / \mathrm{kg})$ solution was injected intraperitoneally. In some experiments, different doses of sodium pyruvate (250 or $1000 \mathrm{mg} / \mathrm{kg}$ ) were given. For tissue examination, brains were harvested at $72 \mathrm{hr}$ after the ischemia, except in cases in which brains were obtained at 15 or $30 \mathrm{~d}$.

Tissue preparation and zinc-specific fluorescence staining. Coronal brain sections (10- $\mu \mathrm{m}$-thick) (anteroposterior, $4.0 \mathrm{~mm}$ from the bregma) were prepared using a cryostat and mounted on prechilled glass slides coated with poly-L-lysine. Unfixed brain sections were stained for $90 \mathrm{sec}$ with the zinc-specific fluorescent dye $N$-(6-methoxy-8-quinolyl)-p-carboxybenzoylsulphonamide (TFL-Zn) (Calbiochem, La Jolla, CA) (Budde et al., 1997; Lee et al., 2000) dissolved in Tris buffer (0.1 mM, pH 8.0). After washing with saline, TFL-Zn-stained sections were examined under a fluorescence microscope (excitation, 355-375 nm; dichroic, $380 \mathrm{~nm}$; barrier, $420 \mathrm{~nm}$ ) (BX60; Olympus Optical, Tokyo, Japan) and photographed with a digital camera (Camedia C2000; Olympus Optical).

Assessment of neuronal death. Morphological changes of neuronal cell death in cortical culture were observed under a phase-contrast microscope (IX70; Olympus Optical). To identify dead neurons, cultures were stained with $0.4 \%$ trypan blue for $3 \mathrm{~min}$. Numbers of trypan blue-stained cells were counted in five randomly chosen fields $(200 \times)$. Percentage of neuronal cell death was calculated by dividing the number of trypan blue-positive neurons by the mean number of neurons in control cultures.

To quantify neuronal cell death in the brain sections, brain sections were processed for terminal deoxynucleotidyl transferase-mediated biotinylated UTP nick end labeling (TUNEL) staining with the in situ cell death detection kit (Boehringer Mannheim, Mannheim, Germany). Briefly, after fixation in $4 \%$ paraformaldehyde and incubation in permeabilization solution ( $0.1 \%$ Triton $\mathrm{X}-100$ in $0.1 \%$ sodium citrate), sections were incubated with TUNEL reaction mixture at $37^{\circ} \mathrm{C}$. The TUNELstained sections were examined under a fluorescent microscope and photographed. Subsequently, the same sections were stained with $0.5 \%$ cresyl violet to evaluate surviving neurons.

TUNEL-positive neurons and surviving neurons with intact morphology on cresyl violet-stained slides were counted bilaterally in the marked areas (see Fig. $3 F$ ) of CA1, CA3, and dentate gyrus with Image-Pro Plus computer-assisted image analysis program (Media Cybernetics, Silver Spring, MD). Statistical analysis were performed by a two-tailed paired $t$ test, and $p<0.05$ was considered statistically significant.

\section{RESULTS}

Before doing experiments in the rat, we determined the spectrum of pyruvate neuroprotection in cortical culture. As reported previously (Sheline et al., 2000), neuronal death induced by $24 \mathrm{hr}$ exposure to $35 \mu \mathrm{M}$ zinc was completely blocked by $5 \mathrm{~mm}$ pyruvate
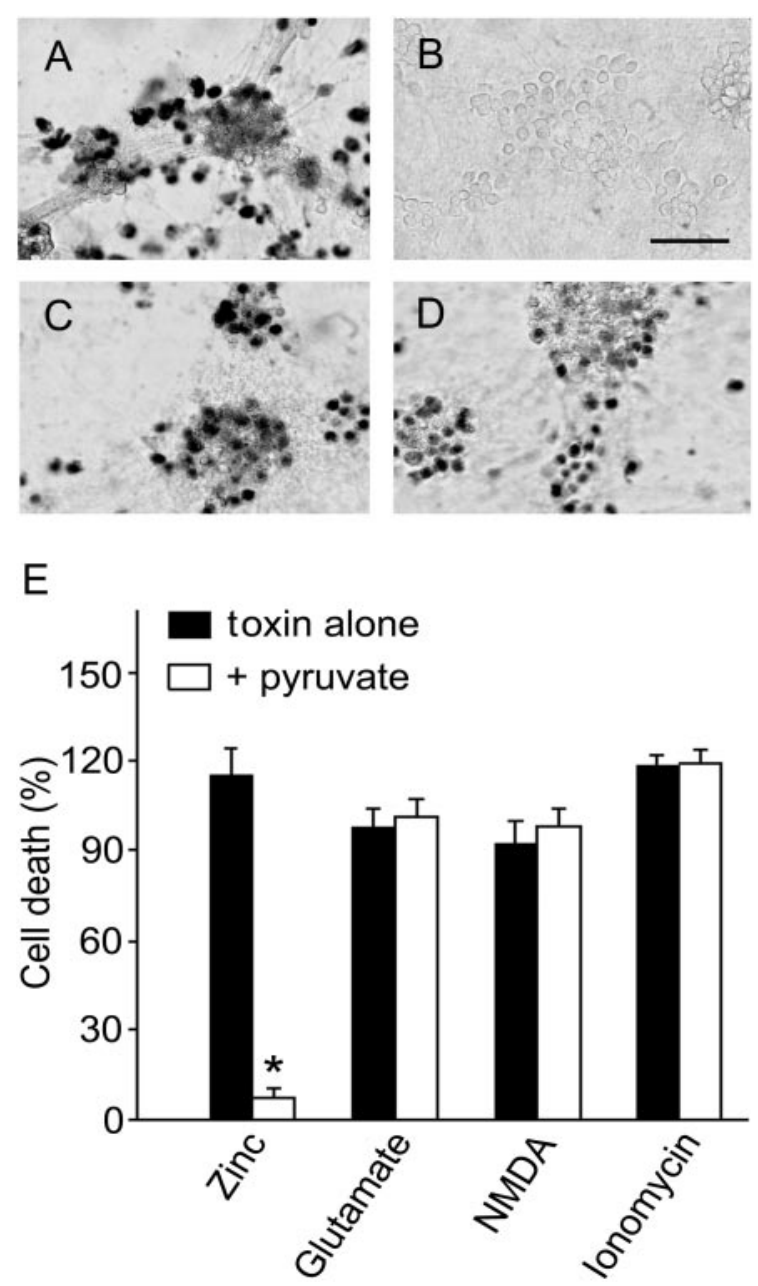

Figure 1. Pyruvate blocks zinc toxicity but not calcium-overload toxicity. $A-D$, Trypan blue-stained cortical cultures after $24 \mathrm{hr}$ exposure to $35 \mu \mathrm{M}$ $\mathrm{ZnCl}_{2}(A, B)$ or $30 \mu \mathrm{M}$ NMDA $(C, D)$ in the absence $(A, C)$ or presence $(B, D)$ of $5 \mathrm{~mm}$ pyruvate. $E$, Bars denote percentage of neuronal cell death (mean $\pm \mathrm{SEM} ; n=4)$ in cortical cultures after $24 \mathrm{hr}$ exposure to zinc, NMDA, glutamate $(100 \mu \mathrm{M})$, or ionomycin $(500 \mathrm{nM})$, alone or with the addition of $5 \mathrm{~mm}$ pyruvate. Asterisks denote difference from toxin alone ( $p<0.01$; two-tailed $t$ test with Bonferroni correction for 4 comparisons). Scale bar, $100 \mu \mathrm{m}$.

in cortical culture (Fig. $1 A, B$ ). However, calcium-overload neuronal death induced by $24 \mathrm{hr}$ exposure to NMDA (Fig. 1C,D), glutamate, or ionomycin was not attenuated by pyruvate (Fig. $1 E$ ). This result indicates that pyruvate is a potent protectant against zinc toxicity but not against calcium-overload toxicity.

Next we examined the effect of pyruvate on transient forebrain ischemia. Transient (12 min) forebrain ischemia was delivered as described in Materials and Methods. Compared with shamoperated rats, rats subjected to ischemia exhibited decreased activity after the surgery. In some, salivation, tremor, startle responses, or wet-dog shakes started to show in $30 \mathrm{~min}$ to $1 \mathrm{hr}$ after ischemia. $\mathrm{NaCl}(104.5 \mathrm{mg} / \mathrm{kg}$, equimolar to $500 \mathrm{mg} / \mathrm{kg}$ sodium pyruvate) or sodium pyruvate $(500 \mathrm{mg} / \mathrm{kg})$ solution was intraperitoneally injected $30 \mathrm{~min}$ after the onset of reperfusion. The $\mathrm{NaCl}$ injection did not alter the behavior of ischemiasubjected rats. In contrast, the pyruvate treatment abolished salivation, tremor, startling, and wet-dog shakes after ischemia, if had been present.

The next conspicuous effect of pyruvate treatment was a re- 


\section{Table 1. Mortality of rats subjected to 12 min forebrain ischemia \\ $\mathrm{NaCl}, 30 \mathrm{~min}$ after the onset of reperfusion \\ 18 of $31(58.1 \%)$ \\ Pyruvate, $30 \mathrm{~min}$ before the onset of ischemia \\ 4 of $9(44.4 \%)$ \\ Pyruvate, at the time of reperfusion \\ 0 of $5(0 \%)$ \\ 1 of $16(6.3 \%)$ \\ 0 of $5(0 \%)$ \\ 4 of $4(100 \%)$ \\ 3 of $7(42.9 \%)$ \\ Pyruvate, $2 \mathrm{hr}$ after the onset of reperfusion \\ Pyruvate, $3 \mathrm{hr}$ after the onset of reperfusion}

Total of 77 adult rats were used for experiments. $\mathrm{NaCl}(104.5 \mathrm{mg} / \mathrm{kg})$ or sodium pyruvate $(500 \mathrm{mg} / \mathrm{kg})$ was intraperitoneally injected at the indicated time in relation to the onset of ischemia or reperfusion.

markable decrease in mortality associated with transient forebrain ischemia. Compared with the high mortality $(58.1 \%)$ of $\mathrm{NaCl}$-injected rats, the mortality of rats injected with sodium pyruvate $(500 \mathrm{mg} / \mathrm{kg})$ at $0-1 \mathrm{hr}$ after reperfusion was $3.8 \%$ (only 1 of 26 rats died) (Table 1). In some rats, sodium pyruvate was injected either $30 \mathrm{~min}$ before or 2 and $3 \mathrm{hr}$ after the onset of reperfusion; in these groups, mortality was 44.4, 100, and $42.9 \%$, respectively (Table 1$)$.

At $72 \mathrm{hr}$ after the $12 \mathrm{~min}$ ischemia, severe neuronal death in hippocampus and cortex was seen in brains of survived $\mathrm{NaCl}$ injected control rats. TUNEL staining showed widespread nuclear DNA breakage in hippocampal and cortical neurons (Fig. $2 A, C)$. The widespread neuronal death was confirmed by cresyl violet staining; degenerating neurons exhibited severe pyknosis (Fig. 2A,C, insets). All of the dead neurons in hippocampus and cortex exhibited dense zinc accumulation in perikarya (Fig. $2 E, G)$. Similarly extensive neuronal death was evident in rats injected with sodium pyruvate $(500 \mathrm{mg} / \mathrm{kg}) 30 \mathrm{~min}$ before reperfusion or $3 \mathrm{hr}$ after ischemia. However, in rats injected with sodium pyruvate $(500 \mathrm{mg} / \mathrm{kg})$ at $0 \mathrm{~min}, 30 \mathrm{~min}$, or $1 \mathrm{hr}$ after the onset of reperfusion, virtually no neuronal cell death was found in cortex or hippocampus (Fig. $2 B, D$ ). In these groups, no zinc accumulation in neuronal cell bodies was seen (Fig. $2 F, H$ ).

Surviving neurons with intact nuclei and no pyknosis were counted in the marked areas (Fig. $3 F$ ) of CA1, CA3, and dentate gyrus in cresyl violet-stained hippocampal sections (Fig. $3 A$ ). To additionally quantify dead neurons, TUNEL-stained neurons were counted in the same areas (Fig. 3B). Twelve minutes of ischemia produced rather severe injury involving all of the areas of hippocampus in the NaCl-treated control rats. However, the same ischemia produced almost no neuronal death in rats intraperitoneally injected with 500 and $1000 \mathrm{mg} / \mathrm{kg}$ pyruvate $30 \mathrm{~min}$ after the onset of reperfusion (Fig. $3 A, B$ ). On the other hand, the $250 \mathrm{mg} / \mathrm{kg}$ dose of pyruvate was modestly protective only in CA3 but not in CA1 or dentate gyrus. At 500 and $1000 \mathrm{mg} / \mathrm{kg}$ doses of pyruvate, cortical neurons were also almost completely protected (data not shown).

Next, the dose of pyruvate was fixed at $500 \mathrm{mg} / \mathrm{kg}$, and the injection time was varied. When administered $0-1 \mathrm{hr}$ after the onset of reperfusion, almost complete protection was seen in all three areas of hippocampus (Fig. $3 C, D$ ). However, when pyruvate was given $30 \mathrm{~min}$ before the reperfusion, only modest protection was seen in CA3 and dentate gyrus but not in CA1. Pyruvate given $3 \mathrm{hr}$ after the onset of reperfusion had no protective effect at all (Fig. 3C,D).

Finally, we examined whether the protective effect of pyruvate was transitory or long lasting. At 15 and $30 \mathrm{~d}$ after $12 \mathrm{~min}$ ischemia, brains of rats given $\mathrm{NaCl}$ or sodium pyruvate (500 $\mathrm{mg} / \mathrm{kg}$ ) injection at $30 \mathrm{~min}$ after the onset of reperfusion and
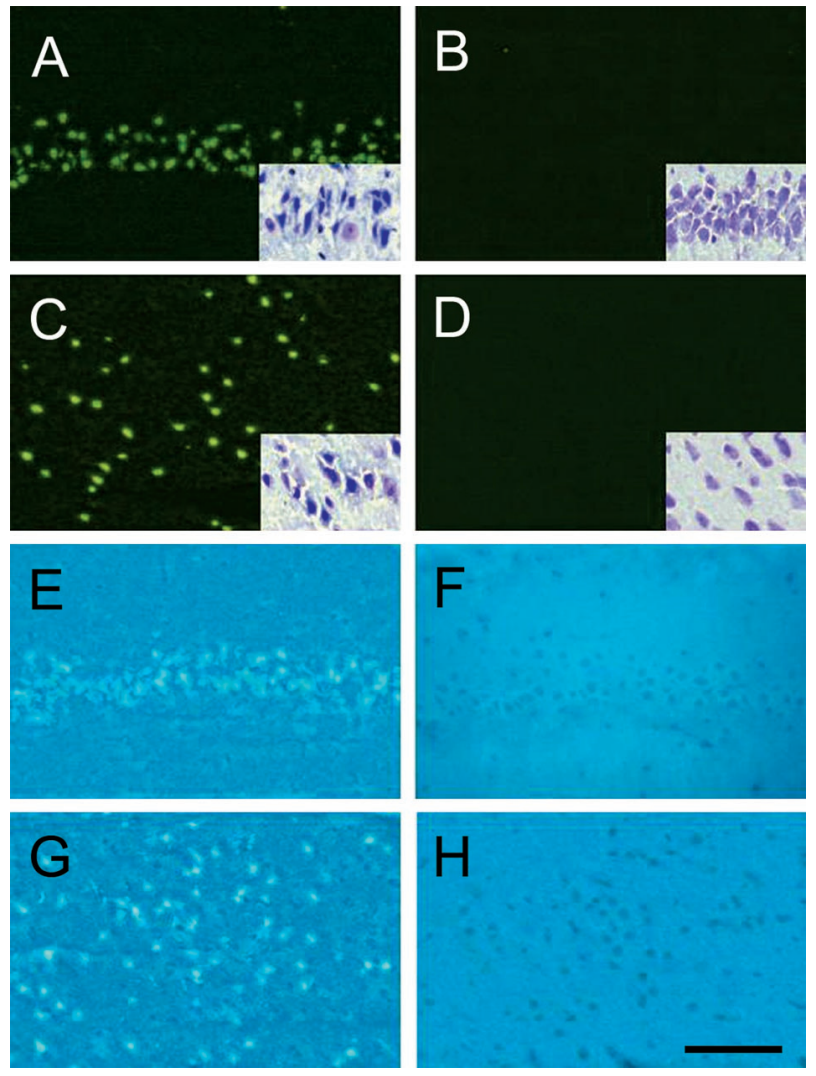

Figure 2. Protection of hippocampal and cortical neurons by pyruvate against transient cerebral ischemia. Hippocampal CA1 $(A, B)$ and parietal cortex $(C, D)$ of rats that underwent $12 \mathrm{~min}$ forebrain ischemia followed 30 min later by intraperitoneal injection of $\mathrm{NaCl}(A, C)$ solution or sodium pyruvate $(500 \mathrm{mg} / \mathrm{kg} ; B, D)$ solution were stained with TUNEL or cresyl violet (insets). Whereas almost complete neuronal damage is evident in $A$ and $C$, no damage is seen in $B$ and $D$. Hippocampal CA1 $(E, F)$ and parietal cortex $(G, H)$ of rats that underwent 12 min forebrain ischemia followed $30 \mathrm{~min}$ later by intraperitoneal injection with $\mathrm{NaCl}(E$, $G$ ) or sodium pyruvate $(500 \mathrm{mg} / \mathrm{kg} ; F, H)$ were stained with TFL-Zn 72 hr after ischemia. Chelatable zinc is visualized as green-blue fluorescence. Dense zinc accumulation in degenerating or dead neuronal cell bodies is seen in NaCl-treated control brains $(E, G)$. No zinc accumulation in neuronal cell bodies is seen in pyruvate-treated cases $(F, H)$. Scale bar, $50 \mu \mathrm{m}$.

were harvested, and the numbers of surviving neurons were counted in the above marked areas (Fig. $3 F$ ) in hippocampus. Because the dead neurons may have disappeared at these time points, surviving neurons in cresyl violet-stained sections were counted. Cell counting revealed that the near-complete neuroprotective effect of pyruvate remained virtually unchanged over the $30 \mathrm{~d}$ time period after the ischemia (Fig. 3E). These data suggest that protective effect of pyruvate is long lasting and likely permanent.

\section{DISCUSSION}

The core finding of the present study is that administration of pyruvate, when given within $1 \mathrm{hr}$ after the onset of reperfusion, is remarkably neuroprotective in rats against transient cerebral ischemia, which produced neuronal death throughout hippocampus. Whereas the overall mortality associated with 12 min forebrain ischemia was $58.1 \%$, pyruvate injection between 0 and $1 \mathrm{hr}$ after the onset of reperfusion drastically decreased it to $3.8 \%$. In addition, the histological protection was so spectacular that no sign of brain damage was found in most of rats. Furthermore, all 
A

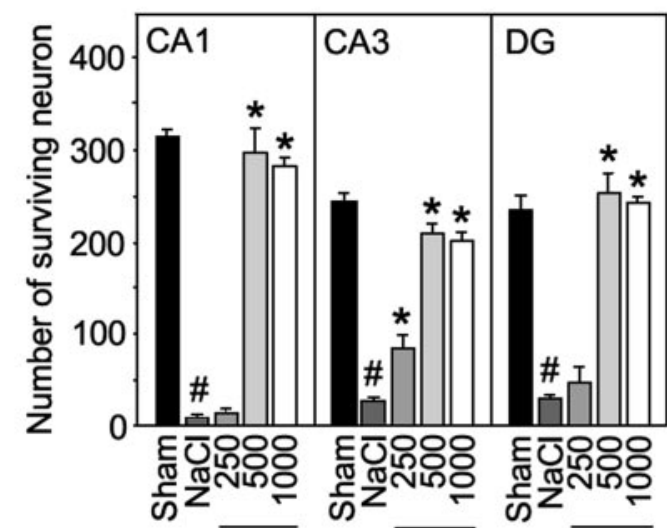

Dose of sodium pyruvate $(\mathrm{mg} / \mathrm{kg})$

C

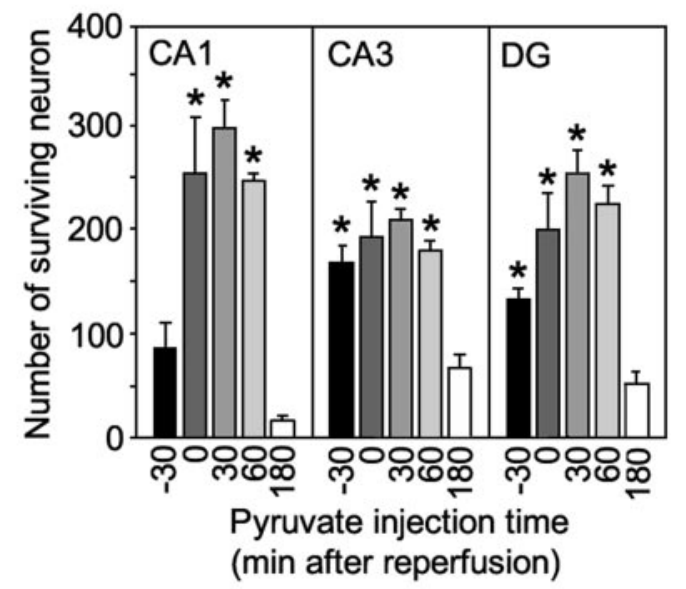

E

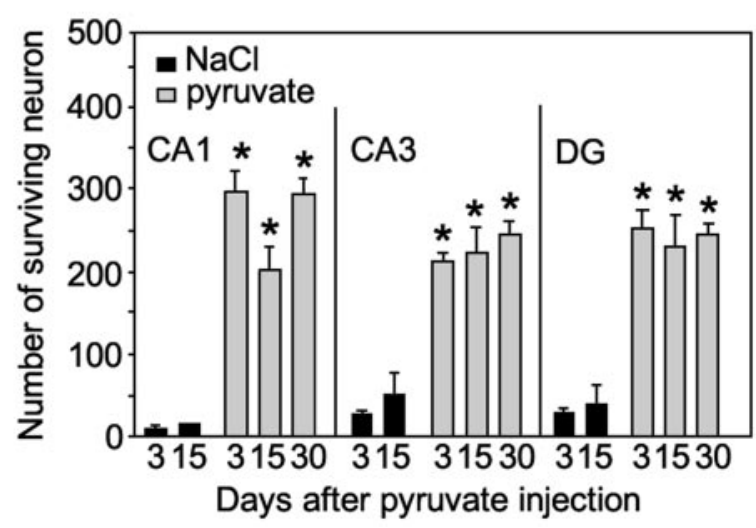

B

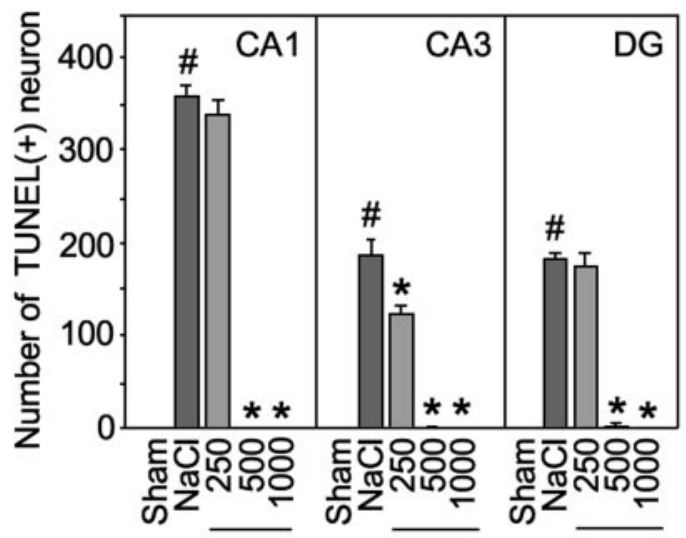

Dose of sodium pyruvate $(\mathrm{mg} / \mathrm{kg})$

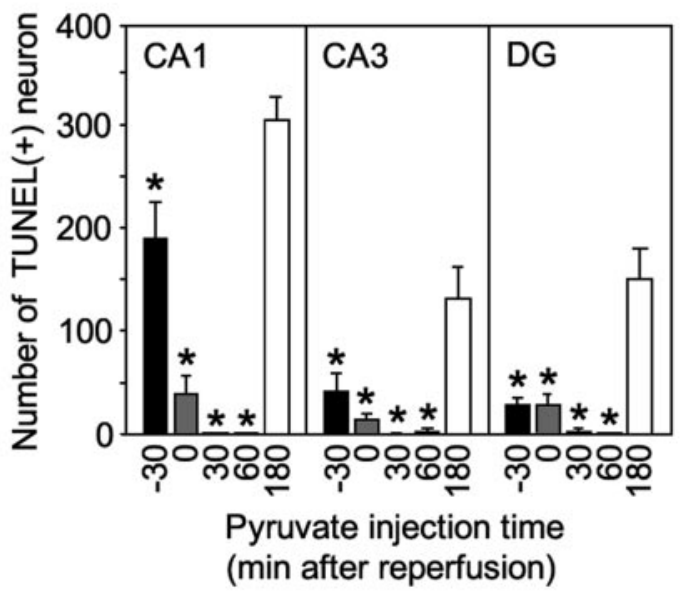

F

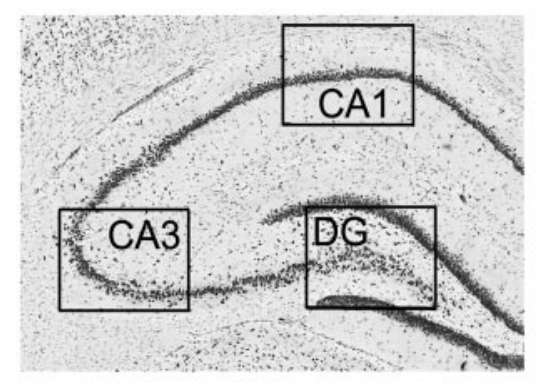

Figure 3. Quantification of pyruvate neuroprotection in hippocampus. A, Surviving neurons were estimated by cresyl violet staining. Bars denote the number (mean $\pm \mathrm{SEM} ; n=5$ each) of intact neurons in each area in sham-operated rats or rats subjected to 12 min ischemia followed by intraperitoneal injections of $\mathrm{NaCl}$ or indicated doses of sodium pyruvate 30 min later. $B$, Dead neurons were estimated by the TUNEL staining in the same sections as in $A$. Bars denote the number TUNEL-positive neurons. Virtually no TUNEL-positive neurons were seen in sham-operated rats or rats given 500 or $1000 \mathrm{mg} / \mathrm{kg}$ sodium pyruvate. $C$, Bars denote the number of intact neurons in rats $(n=5$ each) subjected to 12 min ischemia followed by intraperitoneal sodium pyruvate injections $(500 \mathrm{mg} / \mathrm{kg})$ at $30 \mathrm{~min}$ before or $0 \mathrm{~min}, 30 \mathrm{~min}, 1 \mathrm{hr}$, or $3 \mathrm{hr}$ after the onset of reperfusion. Whereas pyruvate injections at $0-1 \mathrm{hr}$ after ischemia almost completely protected hippocampal neurons, pyruvate injections at 30 min before or $3 \mathrm{hr}$ after the reperfusion protected only minimally. $D$, TUNEL-positive neurons were counted in the same areas of the above rats. $E$, Black bars denote surviving neurons in the marked areas in cresyl violet-stained hippocampi of NaCl-injected rats obtained 15 and $30 \mathrm{~d}$ after 12 min ischemia $(n=5$ each). Gray bars denote surviving neurons in hippocampi of pyruvate-injected rats (30 min after the reperfusion; $500 \mathrm{mg} / \mathrm{kg}$ ) obtained 3, 15, and $30 \mathrm{~d}$ after ischemia. $F$, A cresyl violet-stained hippocampal section of a normal rat. Boxes $(820 \times 615 \mu \mathrm{m})$ denote the areas for cell counting. All counting was performed bilaterally. $A$, $B, \# p<0.001$ denotes difference from the sham-operated groups; two-tailed $t$ test with Bonferroni correction for multiple comparisons. $A-E$, ${ }^{*} p<0.05$ represents differences from corresponding $\mathrm{NaCl}$-injected groups. 
of the pyruvate-rescued neurons seemed to be surviving even $30 \mathrm{~d}$ after ischemia. Naturally, the time window of opportunity for pyruvate protection was not permanent and disappeared when given at 2 or $3 \mathrm{hr}$ after the reperfusion.

The protective time window of pyruvate treatment, $0-1 \mathrm{hr}$ after the onset of reperfusion, is clinically exploitable in the context of cardiac arrest. Whereas resuscitative efforts often restore perfusion to the brain, still a number of patients end up having permanent brain damage, because only minutes of global ischemia can trigger processes leading to permanent brain damage (Pulsinelli et al., 1982; Smith et al., 1984). Hence, if pyruvate proves as remarkably protective in humans as in rats, resuscitation followed by pyruvate treatment within $1 \mathrm{hr}$ would save a number of patients from permanent brain damage. It is not clear why pyruvate given 30 min before reperfusion showed only modest protective effect. This may be attributable to rapid degradation of pyruvate or to the conversion of pyruvate to potentially harmful lactate during ischemia (Petito et al., 1987). In contrast, pyruvate given after reperfusion may be preferentially used in the Krebs' cycle.

Protective doses of pyruvate given intraperitoneally in the present study are $250-1000 \mathrm{mg} / \mathrm{kg}$. Although pharmacokinetic data are not available in this case because pyruvate is rapidly and efficiently transported across the blood-brain barrier (Pardridge and Oldendorf, 1977; Miller and Oldendorf, 1986), these systemic doses may be sufficient to deliver protective concentrations of pyruvate to the brain. Future studies may provide information regarding pharmacokinetics of intraperitoneally injected pyruvate.

Although glutamate antagonists may be effective against ischemic neurodegeneration (Simon et al., 1984; Foster et al., 1988; Judge et al., 1991; Diemer et al., 1992), a significant limitation is that their neuroprotective effect is not long lasting (Colbourne et al., 1999). In contrast, the neuroprotective effect of pyruvate persisted even at $30 \mathrm{~d}$ after the insult, without noticeable side effects.

Whereas the extent and the sustainability of pyruvate neuroprotection in vivo seen in the present study are quite remarkable and surprising, pyruvate neuroprotection per se is not new. Introduction of pyruvate in glucose-deprived neurons restored excitatory postsynaptic potentials and blocked a neuronal damage in hippocampal slice or cultured neurons (Izumi et al., 1994; Matsumoto et al., 1994; Izumi et al., 1997). Moreover, pyruvate has been shown to protect neurons against various insults (Desagher et al., 1997; Ruiz et al., 1998; Maus et al., 1999).

Although elucidation of the mechanism of pyruvate neuroprotection is beyond the scope of the present study, it is likely that normalization of disturbances in energy metabolism may be involved. As Sheline et al. (2000) showed, 4 mm pyruvate normalized ATP and NAD ${ }^{+}$levels in zinc-exposed neurons and protected them against zinc toxicity. Interestingly, our culture data indicate that the protective effect by $5 \mathrm{~mm}$ pyruvate was almost complete in zinc toxicity but not seen in the continuous exposure paradigms of calcium-overload neurotoxicity. However, in a study using a pulse exposure paradigm in the absence of glucose, $5 \mathrm{~mm}$ pyruvate reduced NMDA-induced neuronal death, albeit modestly in striatal cultures (Maus et al., 1999). Without knowing which paradigm is more appropriate in vivo, it appears possible that calcium excitotoxicity may be one of the targets of pyruvate protection. However, the fact that $5 \mathrm{~mm}$ pyruvate almost completely protected against zinc neurotoxicity in our cortical culture seems to strongly favor zinc neurotoxicity as the main target of pyruvate protection in the case of transient cerebral ischemia.

Regardless of the precise protective mechanism of pyruvate against ischemic brain injury, the important fact is that pyruvate is a cheap, easily administrable, innocuous, and most importantly, remarkably neuroprotective substance. Expedient clinical trials seem to be warranted.

\section{REFERENCES}

Aniksztejn L, Charton G, Ben-Ari Y (1987) Selective release of endogenous zinc from the hippocampal mossy fibers in situ. Brain Res 404:58-64.

Assaf SY, Chung SH (1984) Release of endogenous $\mathrm{Zn}^{2+}$ from brain tissue during activity. Nature 308:734-736.

Budde T, Minta A, White JA, Kay AR (1997) Imaging free zinc in synaptic terminals in live hippocampal slices. Neuroscience 79:347-358.

Choi DW (1995) Calcium: still center-stage in hypoxic-ischemic neuronal death. Trends Neurosci 18:58-60.

Choi DW, Koh JY (1998) Zinc and brain injury. Annu Rev Neurosci $21: 347-375$

Choi DW, Rothman SM (1990) The role of glutamate neurotoxicity in hypoxic-ischemic neuronal death. Annu Rev Neurosci 13:171-182.

Colbourne F, Li H, Buchan AM (1999) Continuing postischemic neuronal death in CA1: influence of ischemic duration and cytoprotective doses of NBQX and SNX-111 in rats. Stroke 30:662-668.

Desagher S, Glowinski J, Prémont J (1997) Pyruvate protects neurons against hydrogen peroxide-induced toxicity. J Neurosci 17:9060-9067.

Diemer NH, Jorgensen MB, Johansen FF, Sheardown M, Honore T (1992) Protection against ischemic hippocampal CA1 damage in the rat with a new non-NMDA antagonist, NBQX. Acta Neurol Scand 86:45-49.

Foster AC, Gill R, Woodruff GN (1988) Neuroprotective effects of MK801 in vivo: selectivity and evidence for delayed degeneration mediated by NMDA receptor activation. J Neurosci 8:4745-4754.

Frederickson CJ (1989) Neurobiology of zinc and zinc-containing neurons. Int Rev Neurobiol 31:145-238.

Frederickson CJ, Suh SW, Silva D, Frederickson CJ, Thompson RB (2000) Importance of zinc in the central nervous system: the zinccontaining neuron. J Nutr 130:1471S-1483S.

Howell GA, Welch MG, Frederickson CJ (1984) Stimulation-induced uptake and release of zinc in hippocampal slices. Nature 308:736-738

Izumi Y, Benz AM, Zorumski CF, Olney JW (1994) Effects of lactate and pyruvate on glucose deprivation in rat hippocampal slices. NeuroReport 5:617-620.

Izumi Y, Benz AM, Katsuki H, Zorumski CF (1997) Endogenous monocarboxylates sustain hippocampal synaptic function and morphological integrity during energy deprivation. J Neurosci 17:9448-9457.

Judge ME, Sheardown MJ, Jacobsen P, Honore T (1991) Protection against post-ischemic behavioral pathology by the alpha-amino-3hydroxy-5-methyl-4-isoxazolepropionic acid (AMPA) antagonist 2,3dihydroxy-6-nitro-7-sulfamoyl-benzo(f)quinoxaline (NBQX) in the gerbil. Neurosci Lett 133:291-294.

Kim YH, Kim EY, Gwag BJ, Sohn S, Koh JY (1999) Zinc-induced cortical neuronal death with features of apoptosis and necrosis: mediation by free-radicals. Neuroscience 89:175-182.

Koh JY, Suh SW, Gwag BJ, He YY, Hsu CY, Choi DW (1996) The role of zinc in selective neuronal death after transient global cerebral ischemia. Science 272:1013-1016.

Lee JY, Cole TB, Palmiter RD, Koh JY (2000) Accumulation of zinc in degenerating hippocampal neurons of ZnT3-null mice after seizures: evidence against synaptic vesicle origin. J Neurosci 20:RC79(1-5).

Lobner D, Canzoniero LM, Manzerra P, Gottron F, Ying H, Knudson M, Tian M, Dugan LL, Kerchner GA, Sheline CT, Korsmeyer SJ, Choi DW (2000) Zinc-induced neuronal death in cortical neurons. Cell Mol Biol 46:797-806.

Matsumoto K, Yamada K, Kohmura E, Kinoshita A, Hayakawa T (1994) Role of pyruvate in ischaemia-like conditions on cultured neurons. Neurol Res 16:460-464.

Maus M, Marin P, Israel M, Glowinski J, Prémont J (1999) Pyruvate and lactate protect striatal neurons against $N$-methyl-D-aspartate-induced neurotoxicity. Eur J Neurosci 11:3215-3224.

Miller LP, Oldendorf WH (1986) Regional kinetic constants for bloodbrain barrier pyruvic acid transport in conscious rats by the monocarboxylic acid carrier. J Neurochem 46:1412-1416.

Palmiter RD, Cole TB, Quaife CJ, Findley SD (1996) ZnT-3, a putative transporter of zinc into synaptic vesicles. Proc Natl Acad Sci USA 93:14934-14939.

Pardridge WM, Oldendorf WH (1977) Transport of metabolic substrates through the blood-brain barrier. J Neurochem 28:5-12.

Park JA, Lee JY, Sato TA, Koh JY (2000) Co-induction of p $75^{\text {NTR }}$ and $\mathrm{p} 75^{\text {NTR }}$-associated death executor in neurons after zinc exposure in cortical culture or transient ischemia in the rat. J Neurosci 20:9096-9103. 
Petito CK, Kraig RP, Pulsinelli WA (1987) Light and electron microscopic evaluation of hydrogen ion-induced brain necrosis. J Cereb Blood Metab 7:625-632.

Pulsinelli WA, Brierley JB, Plum F (1982) Temporal profile of neuronal damage in a model of transient forebrain ischemia. Ann Neurol 11:491-498.

Ruiz F, Alvarez G, Pereira R, Hernandez M, Villalba M, Cruz F, Cerdan S, Bogonez E, Satrustegui J (1998) Protection by pyruvate and malate against glutamate-mediated neurotoxicity. NeuroReport 9:1277-1282.

Sheline CT, Behrens MM, Choi DW (2000) Zinc-induced cortical neuronal death: contribution of energy failure attributable to loss of NAD ${ }^{+}$ and inhibition of glycolysis. J Neurosci 20:3139-3146.

Simon RP, Swan JH, Griffiths T, Meldrum BS (1984) Blockade of $N$-methyl-D-aspartate receptors may protect against ischemic damage in the brain. Science 226:850-852.
Smith ML, Auer RN, Siesjo BK (1984) The density and distribution of ischemic brain injury in the rat following 2-10 min of forebrain ischemia. Acta Neuropathol 64:319-332.

Tonder N, Johansen FF, Frederickson CJ, Zimmer J, Diemer NH (1990) Possible role of zinc in the selective degeneration of dentate hilar neurons after cerebral ischemia in the adult rat. Neurosci Lett 109:247-252.

Vogt K, Mellor J, Tong G, Nicoll R (2000) The actions of synaptically released zinc at hippocampal mossy fiber synapses. Neuron 26:187-196. Weiss JH, Sensi SL, Koh JY (2000) $\mathrm{Zn}^{2+}$ : a novel ionic mediator of neural injury in brain disease. Trends Pharmacol Sci 21:395-401.

Wenzel HJ, Cole TB, Born DE, Schwartzkroin PA, Palmiter RD (1997) Ultrastructural localization of zinc transporter-3 (ZnT-3) to synaptic vesicle membranes within mossy fiber boutons in the hippocampus of mouse and monkey. Proc Natl Acad Sci USA 94:12676-12681. 